OPEN ACCESS

Edited by:

Hang Lin,

Central South University, China

Reviewed by:

Lu Zhang,

University of Illinois at Chicago,

United States

Zhenyang $\mathrm{Xu}$,

University of Science and Technology

Liaoning, China

Jeff $F u$,

Infineon Technologies AG, Germany

${ }^{*}$ Correspondence:

Hong $L$

dhace0819@163.com

Specialty section:

This article was submitted to Earth and Planetary Materials,

a section of the journal

Frontiers in Earth Science

Received: 30 July 2019

Accepted: 19 September 2019

Published: 02 October 2019

Citation:

Deng Z, Li H, Wang L, Liu H and Wang Z (2019) Analysis of Remote Displacement Patterns Monitored by

Tiltmeters During Fluid Injection Into Reservoirs. Front. Earth Sci. 7:259.

doi: 10.3389/feart.2019.00259

\section{Analysis of Remote Displacement Patterns Monitored by Tiltmeters During Fluid Injection Into Reservoirs}

\author{
Zhigang Deng ${ }^{1}$, Hong Li ${ }^{1,2 *}$, Longjiang Wang ${ }^{3}$, HongZhi Liu ${ }^{4}$ and Zhenwei Wang ${ }^{5}$ \\ ${ }^{1}$ College of Mining, Liaoning Technical University, Fuxin, China, ${ }^{2}$ State Key Laboratory of Coastal and Offshore Engineering, \\ Dalian University of Technology, Dalian, China, ${ }^{3}$ School of River and Ocean Engineering, Chongqing Jiaotong University, \\ Chongaing, China, ${ }^{4}$ Shandong Urban Construction Vocational College, Jinan, China, ${ }^{5}$ School of Civil Engineering, North \\ China University of Technology, Beijing, China
}

In order to take far-field translational tilts into account during studying the reservoir flow process of $\mathrm{CO}_{2}$ injection, a group of tiltmeters was installed as an array covering an estimate range of ground spreading from the injection well. The measurements were applicable to ground deformations during a fracturing stimulation treatment, a short-term test of $\mathrm{CO}_{2}$ injection into relatively shallow coal seams and a $\mathrm{CO}_{2}$ injection into deep saline aquifer. An attempt to quantitatively model the rational association between reservoir volumetric disturbance underground and its induced corresponding rather slight distortion on surface was presented. It is as well demonstrated how to sufficiently manipulate vertical unstructured and horizontal divergent model numerical discretization, which eventually makes it feasible to study mechanism between reservoir flow and reservoir Geomechanics by full-field reservoir model. The potential algorithms to improve the computational efficiency and actual demand for an ambitious solver to inverse coupling problem are finally discussed. The study proposed the development of a high-efficiency software package fully capable of dealing with reservoir flow and reservoir Geomechanics to implement the history match study not only on a reservoir itself being injected but also including the full field deformation performance up to the costly ground displacement monitoring data.

Keywords: displacement patterns, $\mathrm{CO}_{2}$, gridding technique, numerical discretization, full-field reservoir

\section{INTRODUCTION}

Just like pulse diagnosis that was ever popular in ancient Oriental medicine, tiltmeters as geophysical measure nowadays detect slight tilt signals reflecting rotating displacement differences at several locations covering a portion of boundary, such as ground or borehole opening, where an offset is distant away from down-hole near-field events of an operating reservoir. The precision of tiltmeter to which tilt can be measured is less than 1 part per billion (i.e., less than 1 inch in 16,000 miles). Cooperated with Geomechanical modeling, far-field monitoring can be a useful tool to understand injection advance process and identify possible effects of intensive non-isothermal operations on the structural integrity of subsurface. Enhanced Oil Recovery (EOR) process 
often involves injection of a large volume of fluid, involving remarkable stress and temperature change, which leads to farfield deformation up to mensurable exposure along an opening bound (Dusseault et al., 1993). Thermal recovery of heavy oil, such as Cyclic Steam Stimulation (CSS) and Steam Assisted Gravity Drainage (SAGD), usually significantly changes the volume of bitumen-bearing reservoir, which induces noticeable uneven uplift of overburden. It was observed that heave and subsidence occur simultaneously across one operation site as the front of heated steam moves through reservoir. More extensive use of tiltmeters are being seen now and these tools are being employed in hybrid arrays consisting of microseismic or InSAR images, too. However, only in case of relatively advantageous long-term $\mathrm{CO}_{2}$ injection, InSAR then turns to be a much better alternative when surface deformation reaches the order of centimeters (Rutqvist et al., 2010). In the meanwhile, tiltmeter array yet remains as the unique choice to monitor remote displacements induced by instantaneous short-term $\mathrm{CO}_{2}$ injection. For instance, in Alberta, a surface tiltmeter array detected the ground surface heaves during well fracturing stimulation and $\mathrm{CO}_{2}$ injection into the Ardley coals. During this so-called CSEMP project, tiltmeter measurement recorded that a well fracture stimulation, a 4-day 180-tonne micro-pilot and a 19-day 850 tonne pilot $\mathrm{CO}_{2}$ injections only brought $0.05,0.55$ and $2 \mathrm{~mm}$, respectively, in the maximum ground displacement. Gondle and Siriwardane (2014) installed thirty-six high precision tiltmeters at the field site to monitor ground deformations caused by $\mathrm{CO} 2$ injection, it found that the maximum surface uplift recorded was about $3.3 \mathrm{~mm}$ (0.13 inches). It has gradually turned into a prominent challenge whether the remote displacement monitoring is effective or not to manage reservoir, i.e., effectively in a quantitative way, how to effectively link the far-field deformation patterns with the straining process at depth. At least the latter is fully coupled by complex reservoir dynamics during flow injection.

\section{SUGGESTED PROCEDURE FOR MODELING}

$\mathrm{CO}_{2}$ injection into coal seams as a tight reservoir usually needs fracturing stimulation ahead. As shown in Figure 1, the ground displacements were interpreted from the data monitored by tiltmeters installed as an array covering a disk range of ground surface centered on the drilled injection well. These are about a CO2-ECBM/sequestration multi-well pilot test being sited in Alberta with regard to relatively much shallow lowrank coal seams.

A tiltmeter is an instrument designed to measure very small ground surface changes from the horizontal level. The continuous data were downloaded monthly. It can be seen from (a) that in this particular case a very slight (i.e., the maximum displacement falls into incredible but actually detectable even about $0.05 \mathrm{~mm}$ !) ground valley but with two different low and high uplifts was reasonably caused by a cross-sectional steep-dip fracture open dominantly during stimulation. The fundamental principle of this interpretation is based on an optimal matching using a 3D analytical solution to the elastic deformation field of a half-space due to an internal shear or a tensile fracture (Okada, 1992). These have been accepted as a common practice. Nevertheless, it indeed lacks of sufficient study to prove if the concrete topography of hundreds of sedimentary layers could be completely ignored or seriously simplified, let alone a full-field reservoir model with a broad ground covering tens of thousands of square meters never contains any geological discontinuity. Even if a discontinuity is in active, it does bring abnormal deformation properties from the continuum media assumption.

Meanwhile, a following short term micro-pilot $\mathrm{CO}_{2}$ injection mainly induced a pattern of ground bulges (with the maximum displacement more than tenfold, i.e., over $0.55 \mathrm{~mm}$ for this time) like a camel's hump with the one occurring to the injector well in the northwest and the other the rough south, respectively. The similarity in shape existing between Figures 1A,B, i.e., the interpretations of remote displacement monitoring on these two different tests both tell a somewhat a pair of camel's hump like surface heave distribution roughly along northwest and southeast, this consistency in pattern most probably implies that the pore pressure ballooning effect in $\mathrm{CO}_{2}$ injection obeyed the same mechanism as that in fracturing fluid (water and sands in this case) in fracture stimulation.

A few years before, double-lobe ground uplift pattern had been observed by satellite-based interferometry (InSAR) at injection well KB-502 in Salah $\mathrm{CO}_{2}$ storage operation at the Krechba gas field, Algeria. In retrospect, almost even a little earlier, Lawrence Berkeley National Laboratory (LBNL) had studied this similar phenomenon by coupled modeling using TOUGH2 and FLAC3D (Rutqvist et al., 2011). It concluded that the doublelobe data correlates with underground reservoir structures, i.e., strongly heterogeneous permeability and deflation of an existing fracture zone or fault near this injection well. Unfortunately, at that time due to lacking of any world's cutting-edge efficient coupling simulator as available tool, only conventional technical work routine was adopted in the history matching study. Shortterm $\mathrm{CO}_{2}$ injection ( $\mathrm{Li}$ et al., 2009) was simulated based on a reservoir model not explicitly including the stimulated fracture in it. As a matter of fact, till now, the most sophisticated workflow on coupled reservoir flows and reservoir Geomechanics using TOUGH2 plus FLAC3D is exclusively grasped by a few scientific and technical entities, i.e., LBNL as well as a few its collaborative companies and academic followers. It is not a universal routine at all. Otherwise, more precise study endeavor might have been able to more reasonably, confidently and quantitatively single out sorption effect in a pure mechanical view (i.e., matrix swelling and softening as known so far) during $\mathrm{CO}_{2}$ injection.

Obviously, the interpretation through optimal matching based on three-dimensional closed-form fundamental solution to an elastic half space acted by an internal open fracture should be restrictedly only applicable to normal or regularly sedimentary underground stratigraphic configurations because it came down to the postulation where homogenization method must stand. Considering the complexity usually existing in underground stratigraphic configurations, especially in the case shown in Figures $\mathbf{2 A , B}$, detailed numerical simulation is helpful and as 


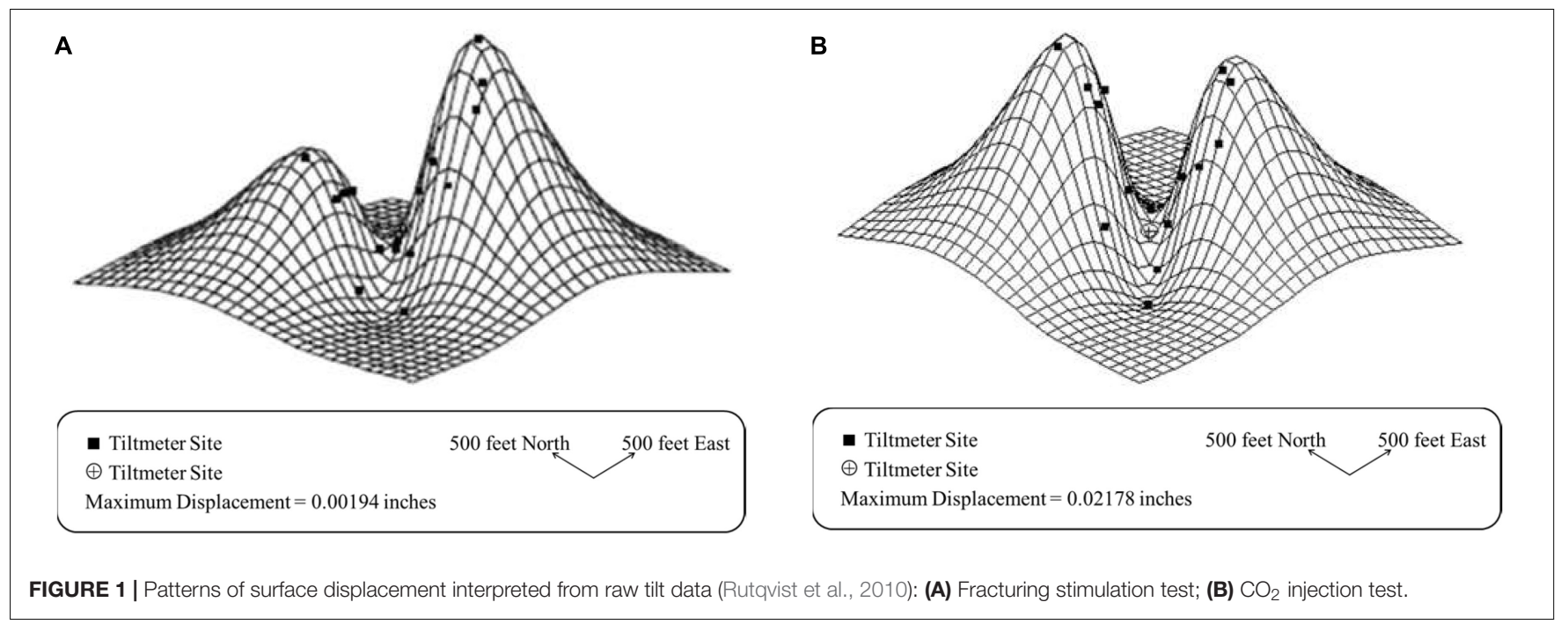

A



B

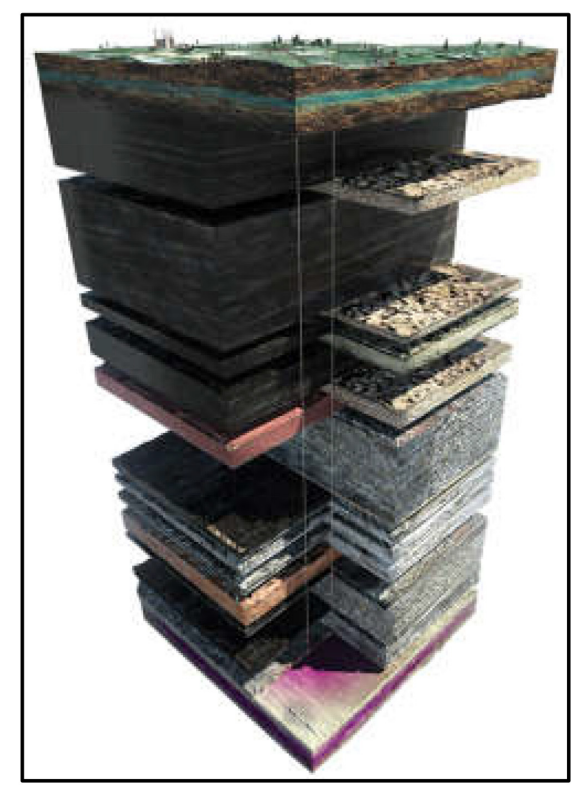

FIGURE 2 | Schematic illustration of different stratigraphic configurations in view of the extent of applicability of homogenization: (A) Metamorphic folding faulting (John Perez Graphics and Design LLC, 2018); (B) Regular sedimentary strata over 3 km down near Estevan, Canada (Aquistore, 2019).

a matter of fact unavoidable to study the data from remote displacement monitoring. As long as the values of comprehensive properties adopted in numerical analyses, which are the elastic constants averaged in a stratum-thickness weighted way for an unified media overall, should be carefully checked or calibrated to see if they also fall consistent with those cases to take lithology description columns on corresponding to well log interpretation, especially the one, either Young's modulus or Poisson ratio, which is found much more sensitive after implementing a careful sensitivity study.

As shown in Figure 3, a dominant state among fracturing process is studied by forwarding simulation using both planestrain and 3D model. In order to correctly represent a fracture, these models need a skill to setup an interface structure and the net pressure is used to apply to open it. The results on surface displacements show plane model turns to relatively overestimate the response though the abruptly slant fracture viewed from its vertical cross section which is interpreted from tiltmeter array detected data and also verified by downhole tilt results has a prominent aspect ratio, i.e., its height from 30 to $50 \mathrm{~m}$ verses its full length more than $300 \mathrm{~m}$.

It is noteworthy that principally this fracture supposes to propagate along the major principal stress within almost horizontal section whose inclination is rather slight as shown in Figure 4A. This might be consistent with the direction of bedding cleats developed in coal so as to conform to the probable 

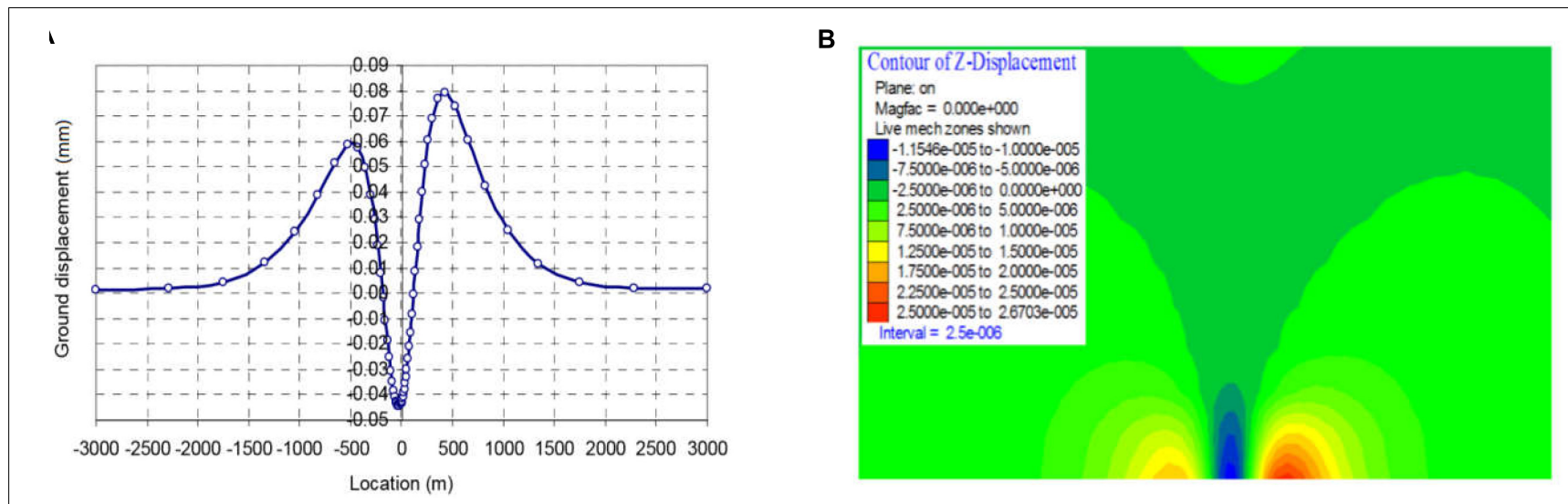

FIGURE 3 | Simulated ground torsion induced by fracturing (Li and Li, 2010): (A) Using Itasca's FLAC ${ }^{2 D \oplus}$; (B) Using Itasca's FLAC3D'
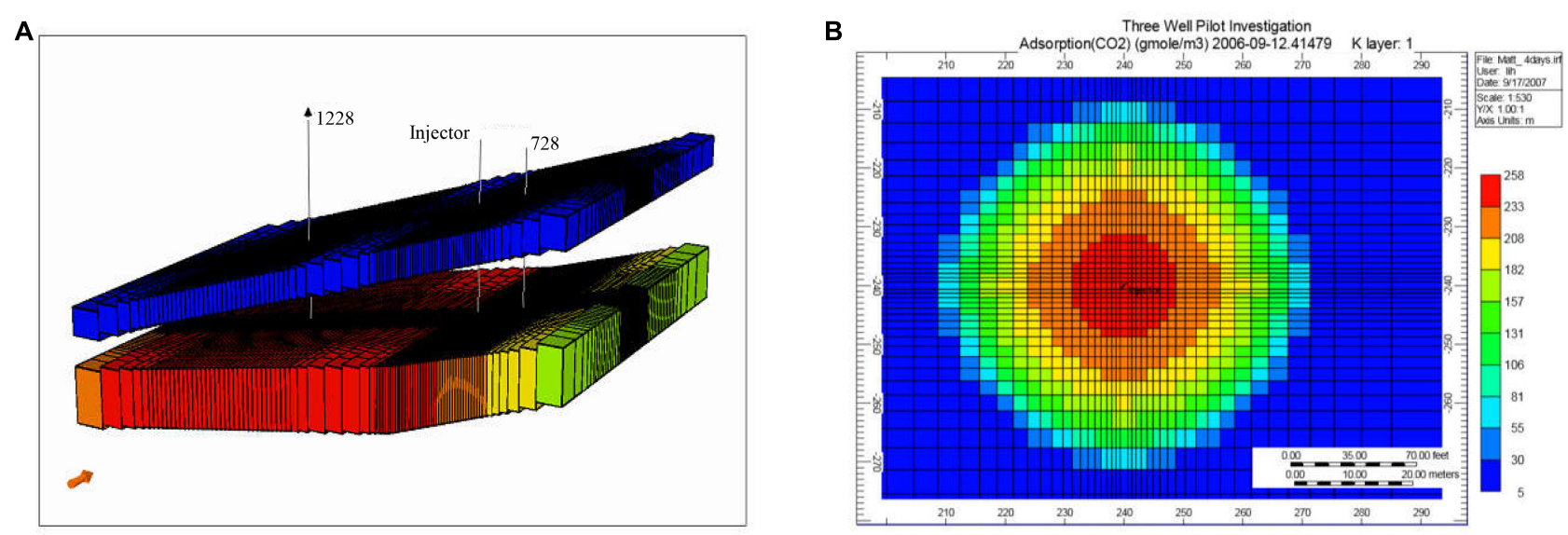

FIGURE 4 | Illustration of history matching simulation on an ECBM testing using CMG's $\mathrm{GEM}{ }^{\oplus}$ : (A) Contour of $\mathrm{CO}_{2}$ concentration adsorbed in coal matrix; (B) Model grids for coal seams in 3D view.

anisotropy in strain associated as well as hydraulic characteristics. Unfortunately, there was then no such detailed study whose necessity had been commonly recognized by cooperating parties. As shown in Figure 4B, the anisotropy brought by either a local fracture or distributive cleat structure hasn't been considered in the study due to shortage of relevant data but also no quantitative information. Further research interests yet exist at including anisotropic porosity and permeability sensitivity to initial stress state and secondarily induced stress concentration.

To model ground heave induced by $\mathrm{CO}_{2}$ injection into coal seams, it is assumed that the volumetric strain corresponding to matrix swelling caused by gas component adsorption is proportional to its adsorption concentration there. It could be reasonably singled out through a sampling experiment through helium injection in the laboratory for the sake of subtracting grain compression effect as the rare amount of helium adsorbed into coal is ignorable (Mazumder et al., 2006). So is its brought swelling. Considering the competition existing mainly between $\mathrm{CO}_{2}$ and $\mathrm{CH} 4$, the effect corresponding to the latter either at reservoir initial condition or during when each component falls into a distinctly partially saturated state would bring significant accuracy loss. Not only matrix swelling caused by gas composition, either each pure component or their composite effect along the whole course of in situ fluid pressure and total stress evolution, but also matrix softening should in the meanwhile be carefully measured.

More careful further research interest yet exists at behavior of the stimulated fracture during $\mathrm{CO}_{2}$ injection into coal seams though this is to intertwine near-field complicated coupled mechanism between reservoir flow and Geomechanics. As a matter of fact, a relatively shallow reservoir $\mathrm{CO}_{2}$ injection which cannot take advantage of supercritical $\mathrm{CO}_{2}$ properties might have met compressed liquid $\mathrm{CO}_{2}$ transiting to gas $\mathrm{CO}_{2}$ in the near field. It is well known that phase transition would bring about distinct heat swallow and spit phenomenon. However, it is completely unknown how this near-field temperature dramatic change influences the near field seepage characteristics. This advocates a non-isothermal multiphase multi-component integrated coupling simulator specially sharpen to $\mathrm{CO}_{2}-\mathrm{ECBM}$ study and design endeavors in the near future. 
It can be seen from the above that to simulate a certain state of rather slight ground surface uplift caused by injection at reservoir depth looks hard to achieve a credible accuracy. Nevertheless, it doesn't mean all endeavor pursuing a quantitative analysis desperately falls into a trivial destiny. Remote displacement as a monitoring means, performs just like blood pressure pulse at one's wrist reflecting the heart rate, even health or a female's pregnancy. Rather than matching its absolute magnitude, the actual significance at studying remote displacements through numerical modeling lies more in matching its distributive patterns over a boundary. Furthermore it lies in matching a series of consecutive states under which it evolves to imply the injection process at depth.

\section{GRIDDING TECHNIQUE TO DEAL WITH FULL-FIELD RESERVOIR}

In order to take far-field displacement into account during simulating $\mathrm{CO}_{2}$ injection into a deep saline aquifer, how to control a compatible numerical discretization was sufficiently studied in modeling from reservoir flow to its induced deformation up to the ground surface. A full-field reservoir model means not only its middle portion as defined in the traditional concept of a reservoir between a depth interval where petroleum resources are contained but also a large scales of both overburden and overlying strata representing distant deformation effects of interest caused by reservoir flows. It is a huge challenge to the efficiency of gridding technology. Earlier pioneers in reservoir Geomechanics have to study simulation grids by themselves to deal with consultant projects (Aziz et al., 1993). Aziz et al. (1993) pointed out that in field-scale applications the need to satisfy a variety of constraints that often are conflicting makes grid generation difficult and timeconsuming. Naturally, one first of all senses a need to extend conventional reservoir model but the work will never grow up so easy. Relatively fine grids focusing on near-field behavior are not consistently necessary to model far field effects. In order to economically use grids, to adopt unstructured meshing between layers usually gets unavoidable. Furthermore, the grids forms a focusing core that captures $\mathrm{CO}_{2}$ plume so as to grow along with $\mathrm{CO}_{2}$ injection progress but also its surrounding transient periphery where radiating mesh gets proportionally coarser and coarser in size. This gridding dynamics must already realize its mature automation.

Therefore, to feasibly discretize a full-field reservoir model lies in two challenges: (1) Once unstructured meshing is adopted across between layers along vertical direction, an interface will be differently represented. Coplanarity throughout this originally common surface may become a contradictory problem because it defined by finer grids from the one side but coarser from the other; (2) Within each layer, grids dynamically evolve to automatically capture $\mathrm{CO}_{2}$ expanding, for an instance. However, the transient 'diffusing' of mesh should keep independent with its defined topography of this layer. The latter is fixed though its meshing keeps changing. It is just like one's photo should be identifiable no matter high or low resolution is adopted to form its bitmap. As shown in Figures 5A,B, the above difficulties had been overcome by successfully realizing secondary development applicable to the existing simulators.

Through similar technology, an identical model gridding was kept between reservoir flow simulation and its associated Geomechanics modeling. The interaction between flows within the reservoir and the deformation of both overburden and underlying strata was so far decoupled as one-way coupling strategy. That is: the independency of reservoir flows is first postulated so a conventional history matching study was implemented; then by assuming the alteration of effective stress should be relatively small enough than those of pore pressure and total stress, the reservoir flows only affect far field strains once. Actually, the most efficient model gridding might be a cylindrical column shape with a circular heart. Further, this technique lays a cornerstone to make the dream become realistic in the future work that the automatic re-meshing focuses so as to capture the spreading area of injected pressure in a dynamical way.

In addition to thousand lines of source codes have been well written and verified in Fortran modules to realize the aforementioned functionalities, e.g., definition of geometry in different resolutions or ranges, in coarse or fine, refined or
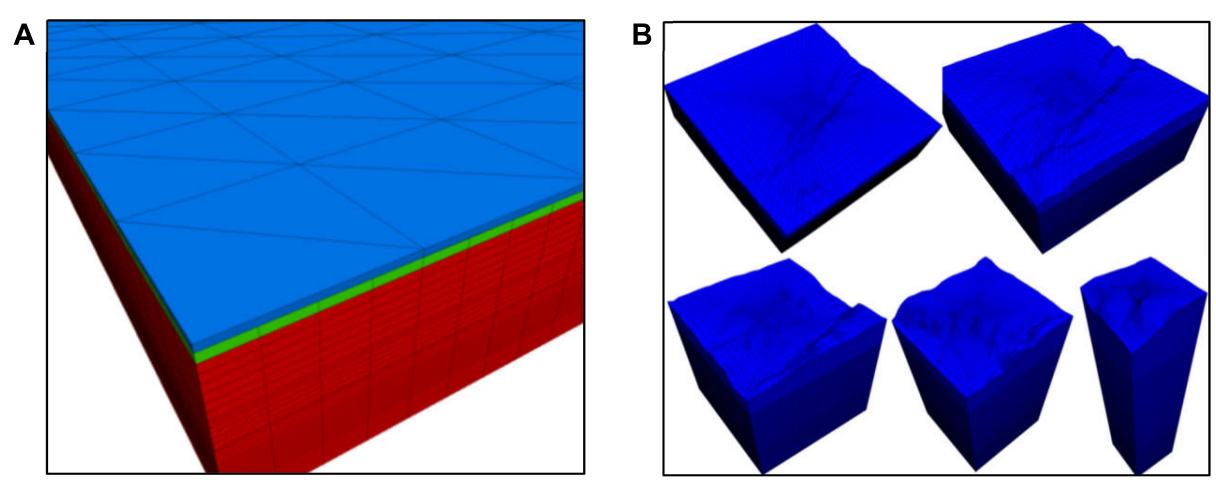

FIGURE 5 | Programmatic gridding technique for advanced full-field reservoir modeling (Li et al., 2015): (A) Each 16 hexahedrons at lower layer is rigidly attached to a pair of triangular prisms; (B) No matter with sparse or dense mesh, its defined topography changes. 
radiant grids, keeping grids independent strata topology between different software through interpolation and extrapolation algorithms, accessory programs have as well been developed to assist in setting up layer dependent property distribution and applying initial and boundary conditions to complex shapes defined by grids. Some procedures must be implemented taking advantage of imbedded language FISH. As repetitively using FLAC3D commands for a large number of times, it would become extremely time-consuming. Furthermore, to carefully compute on one-way coupling reservoir Geomechanics, grids sometimes need to be kept exactly the same or at least consistent between different simulators e.g., CMG's GEM for reservoir flow and Itasca's FLAC3D. Their models are following completely different geometrical data formats.

It is still unknown whether and how the practical winding topography of even relatively flat sedimentary layers or structure of discontinuity over and under an injected reservoir contributes to the ground surface displacement pattern. Further careful study is obviously needed to make this problem clear. As shown in Figures $\mathbf{6 A , B}$, the forwarding simulation on vertical surface displacement as an example for full-field reservoir modeling was smoothly implemented; though underlying strata in general behave with a higher average Young's modulus and lower Poisson ratio, the study demonstrated that enough depth even up to the scale from reservoir over to surface of underlying strata should be included in the full-field reservoir model as the elastic base underneath reservoir contributes its rebounding effect to the surface displacement.

Currently, it still lacks a unified commercial software package based on fully or partially coupling solvers as an efficient tool to analyze the remote slight displacement as well as its pattern evolution induced by the reservoir flow process during fluid injection. Actually, considering the high-accuracy tiltmeter monitoring data is very costly, a high-efficient software package should be urgently proposed and specially developed to simulate reservoir injection coupling process by forward or inverse modeling. It is believed that many reservoir engineers and research scientists desire to do history match study not on reservoir only but also including the full field deformation behavior up to the ground displacement monitoring data.

\section{PURSUING SUPER COMPUTING SOLUTION TO COUPLED/INVERSE PROBLEM}

Schlumberger incorporated industry-reference reservoir simulator Eclipse with an integrated environment for $4 \mathrm{D}$ geomechanics modeling and reservoir simulation - Petrel Reservoir Geomechanics. Meanwhile, Baker Hughes synergized unified finite element analysis Abaqus which simulates realistic performance with advanced multi-physics solutions with comprehensive platform JewelSuite GeoMechanics which improves reservoir insight and accurately assesses geomechanics. Although these jumbos seem to provide possible workflows to simulate reservoir fluid injection process from somewhat coupling point of view, their efficiency is still far less enough to undertake any history match study on the evolution of induced remote displacement on basis of a complex reservoir model. The stratum topography and property variation included in so large a full-field scale, i.e., typical reservoir plus all overburden and same order of scale of underlying layer groups, consumes tremendous nodes and zones even for the minimum numerical resolution, let alone iteration loops unavoidably make the computation plod as reservoir flows induced overburden rock strain is after all a coupling problem.

In order to overcome computational effort, three dimensional boundary element method which alleviate the massive domain

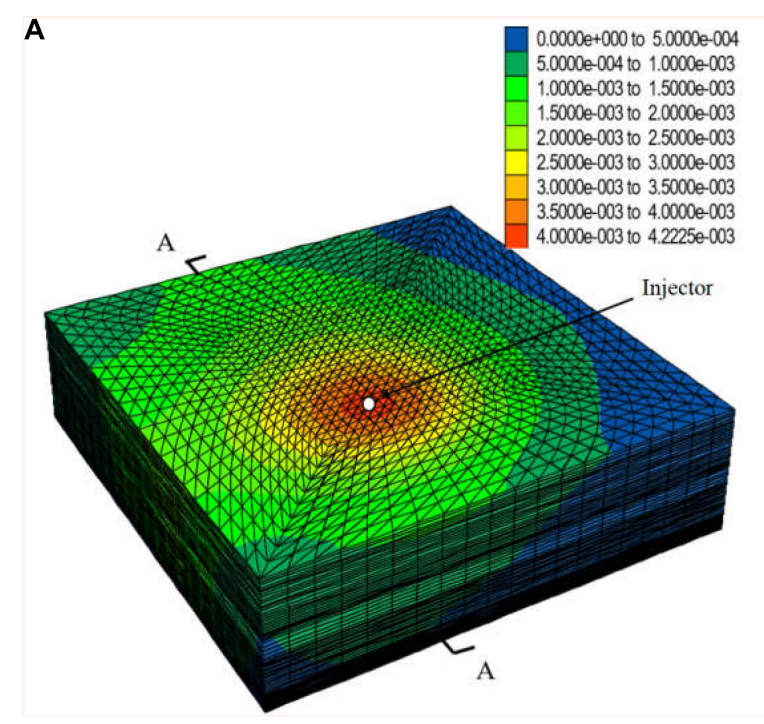

B

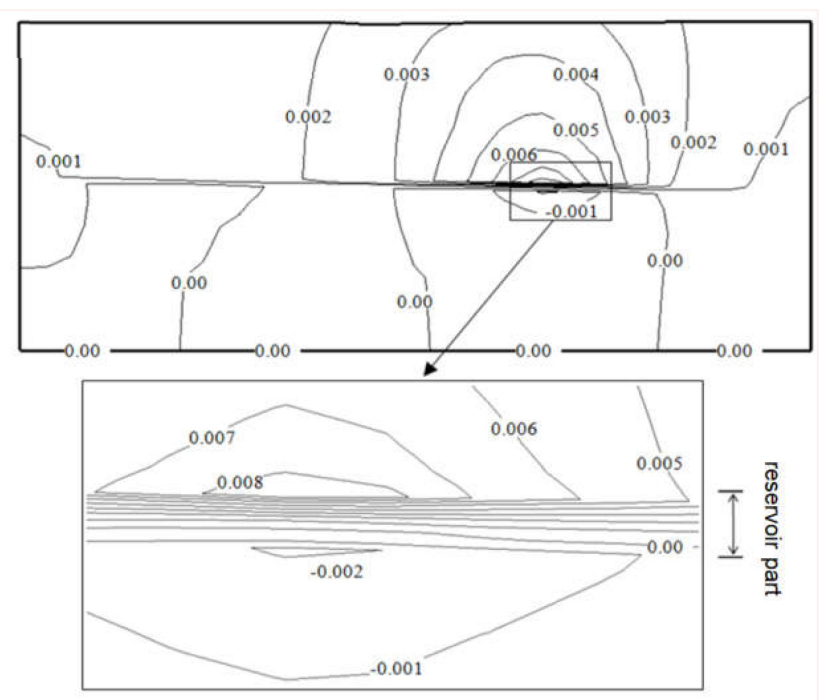

FIGURE 6 | Simulated Z displacement (m) after 1096-day injection of $330 \mathrm{kT} \mathrm{CO}_{2}$ at a project site (Li et al., 2017): (A) Diagram of simulated vertical displacement; (B) Vertical displacement contour along cross section A-A. 
discretization up to only that of the boundaries embracing each stratum group, should be at least introduced to simulate far-field elastic response within this so-called Geomechanics-Reservoir modeling (Yin et al., 2006). Boundary element method generally also improves computational accuracy. Furthermore, it is after all the ground surface tilts that can be measured as known data but the injection pressure distribution inside reservoir is unknown. An ideal analysis shouldn't stop as a try of forwarding simulation but to seek a sensitive solution to this inverse problem based on the principles of optimization as well as spatial regularization (Zabaras et al., 1989).

\section{CONCLUSION}

Ground surface deformation is a security significant geomechanical response during gas injection into a reservoir as it reflects repository inflation as well as its near-field integrity. It should be analyzed with enough precision through numerical modeling to guide tilt-meter array design but also accordingly match monitoring data in a dynamical way.

Containing strata from a reservoir base in depth to overloading rocks till ground surface, an extensive model to study reservoir far-field effects is usually made of more than a hundred of topographical layers. A new efficient gridding approach has already been developed in order to flexibly focus on reservoir pressure alteration area so as to capture ground surface heave with better speed and precision. To follow reservoir pressure growth, horizontal discretization which consists of a stepwise enlarging fine core surrounded by gradually coarser periphery is successfully realized. This technique was well applied and rigorously verified to analyze ground surface deformation induced by carbon dioxide spreading in the deep saline aquifer during a long injection process. A new gridding approach is developed and provided: Considering the feature of our model, in vertical cross section, we can cut flat planes easily but in horizontal direction, we have zigzag topography of much over a hundred strata. In horizontal view, it has a flexible moving core with enough fine grids to focus on pore pressure alteration brought by $\mathrm{CO}_{2}$ plume. Then in order to conveniently set up boundary conditions, the coral part must be surrounded by a periphery part which is proportionally being enlarged grids to form straight plane boundaries parallel to coordinate directions. To implement a controllable flexibly gridding technique, we must have a qualified interpolation algorithm. As grids may be freely changed during preliminary trail study and formal model building but its defined strata morphology must keep unchanged, consistent or independent through the procedures.

The Fortran programmed flexible gridding definition technique is expected to own its further automation and more idealized focused shape so as to capture pressure spreading of injected $\mathrm{CO}_{2}$ plume in a dynamic way. Additionally, the Fortran programming has been utilized to constitute a power user interface to transform the data formats from a reservoir flow simulator into a geomechanical modeling package. The technique is necessary to keep models and meshes identical between reservoir flow and reservoir Geomechanics simulations so as to facilitate the one-way or even iterative coupling solution. Fortran programming is but also very helpful to distribute properties, setup initial conditions and apply boundary conditions in an efficient way. In some cases, due to a large number of repetitive command lines become too costly to computer capacity, Fortran programming gridding control technique must be used in conjunction with FISH language in FLAC3D.

As in general a reservoir flow or Geomechanics simulation professional used to take advantage of the gridding functionality already provided by commercial software packages, the work described in this paper offers readers an exploration for practice of programmatic gridding technique applicable to advanced reservoir Geomechanics modeling. It is expected to be systematically consolidated and further carried forward.

Considering the huge model scale in implementing a fullfield reservoir history matching study which containing the overloading part up to ground surface displacements and the underlying part taking re-bouncing effect into effect, possible complexity of stratum units and decoupling iteration as well as optimization in inverse solution, all demands tremendous computational resources for analyzing remote displacement patterns monitored by tiltmeters during fluid injection into reservoirs. In order to analyze ground surface displacement monitoring in an efficient way and promote the utilization of potential value in tiltmeter array data, a competent numerical tool is strongly recommended to be developed base on a super computing platform in the near future.

\section{DATA AVAILABILITY STATEMENT}

All datasets generated for this study are included in the manuscript/supplementary files.

\section{AUTHOR CONTRIBUTIONS}

ZD and HL conceived the main conceptual ideas. ZD wrote the first draft of the manuscript. LW, HL, and ZW performed some of the data analysis and as well as discussing the results. All authors discussed the results, commented on the manuscript, and provided critical feedback.

\section{FUNDING}

This research was supported by the National Key Research and Development Plan of China (2017YFC1503103), the National Natural Science Foundation of China (Grant No. 51774064), the Natural Science Foundation of Henan Province (182300410161), Excellent Research Team Fund in North China University of Technology (Grant No. 107051360019XN134/017), and Scientific Research Fund in North China University of Technology (Grant No. 110051360002). 


\section{REFERENCES}

Aquistore (2019). Available at: http://aquistore.ca/ (accessed June 9, 2019).

Aziz, K. (1993). Reservoir simulation grids: opportunities and problems. J. Pet. Technol. 45, 658-663. doi: 10.2118/25233-pa

Dusseault, M. B., Bilak, R. A., and Rothenburg, L. (1993). Inversion of surface displacements to monitor in situ processes. Int. J. Rock Mech. Min. Sci. Geomech. Abstr. 30, 1219-1222. doi: 10.1016/0148-9062(93)90098-x

Gondle, R., and Siriwardane, H. (2014). "Field monitoring and modeling of CO2 injection into an unmineable coal seam," in Proceedings of the 48th US Rock Mechanics/Geomechanics Symposium, ARMA, Morgantown.

John Perez Graphics and Design LLC (2018). Available at: https://www.geoart.com/ schematiccategories/onshore/page/2/ (accessed June 9, 2019).

Li, H., and Li, G. (2010). "Modeling surface heave induced by hydraulic fracturing stimulation and CO2 injection into coal seams," in Proceedings of the 44th US Rock Mechanics Symposium and 5th U.S.-Canada Rock Mechanics Symposium, Salt Lake City.

Li, H., Zambrano, G., and Gunter, B. (2009). "Modelling to infer induced ground surface heave during continuous CO2 injection into coal seams," in Poster Sessions of 8th Annual Conference on Carbon Capture and Sequestration, Pittsburgh.

Li, H., Zheng, L. N., Lee, S., and Chalaturnyk, R. (2015). "Application of programmatic gridding technique to advanced reservoir Geomechanics modeling," in Proceedings of 49th U.S. Rock Mechanics/Geomechanics Symposium, San Francisco, CA.

Li, H., Zheng, L. N., and Liu, J. J. (2017). Full-field modeling and analysis of surface deformations induced by gas injections into reservoirs. J. Southwest Pet. University 39, 147-157.

Mazumder, S., Karnik, A., and Wolf, K. H. (2006). Swelling of coal in response to CO2 sequestration for ECBM and its effect on fracture permeability. Soc. Pet. Eng. 11, 390-398. doi: 10.2118/97754-pa
Okada, Y. (1992). Internal deformation due to shear and tensile faults in a half-space. J. Bull. Seismol. Soc. Am. 82, 1018-1040.

Rutqvist, J., Liu, H.-H., Vasco, D. W., Pan, L., Kappler, K., and Majer, E. (2011). Coupled non-isothermal, multiphase fluid flow, and geomechanical modeling of ground surface deformations and potential for induced micro-seismicity at the In Salah CO2 storage operation. Energy Procedia. 4, 3542-3549. doi: 10.1016/j.egypro.2011. 02.282

Rutqvist, J., Vasco, D. W., and Myer, L. (2010). Coupled reservoir-geomechanical analysis of $\mathrm{CO} 2$ injection and ground deformations at in Salah. Algeria. Int. J. Greenhouse Gas Control 4, 225-230. doi: 10.1016/j.ijggc.2009. 10.017

Yin, S., Rothenburg, L., and Dussealt, M. B. (2006). 3D coupled Displacement Discontinuity and Finite Element analysis of reservoir behavior during production in semi-infinite domain. Transp. Porous Media 65, 425-441. doi: 10.1007/s11242-006-0003-2

Zabaras, N., Morellas, V., and Schnur, D. (1989). Spatially regularized solution of inverse elasticity problems using the BEM. Commun. Appl. Numer. Methods 5, 547-553. doi: 10.1002/cnm.16300 50808

Conflict of Interest: The authors declare that the research was conducted in the absence of any commercial or financial relationships that could be construed as a potential conflict of interest.

Copyright (c) 2019 Deng, Li, Wang, Liu and Wang. This is an open-access article distributed under the terms of the Creative Commons Attribution License (CC BY). The use, distribution or reproduction in other forums is permitted, provided the original author(s) and the copyright owner(s) are credited and that the original publication in this journal is cited, in accordance with accepted academic practice. No use, distribution or reproduction is permitted which does not comply with these terms. 